\title{
MASERS ASTROFÍSICOS: I - SOLUÇÕES DA EQUAÇÃO DE TRANSFERÊNCIA RADIATIVA
}

\author{
GILBERTO C. SANZOVO \\ AMAURY A. ALMEIDA \\ PATAN D.SINGH
}

\section{RESUMO}

Masers astrofísicos são fontes em que o coeficiente de absorção da correspondente linha espectral é negativo e a radiação é amplificada ao invés de ser absorvida. Nas fontes não saturadas a amplificação é exponencial. Para as fontes saturadas ela pode crescer linearmente com a distância ou então, com o cubo do comprimento de saturação do maser.

PALAVRAS-CHAVE: Masers astrofísicos; Fontes saturadas; Fontes não saturadas; Transferência radiativa; Meio interestelar.

\section{1 - INTRODUÇÃO}

Um maser* astrofísico é uma fonte de emissão de radiação em microondas que apresenta uma população invertida, atribuída a transições rotacionais ou vibracionais de moléculas situadas nessas fontes. Essa inversão de população é alcançada por meio de um eficiente mecanismo de bombeamento que faz com que a emissão estimulada exceda a absorção. $O$ coeficiente de absorção da correspondente linha espectral é negativo o que implica na amplificação da radiação que atravessa a fonte astrofísica.

As pesquisas sobre os masers astrofísicos começaram quando Weaver et al. (1965) e Gundermann (1965) observaram intensa emissão do radical oxidrila $(\mathrm{OH})$ na direção da nuvem de Orion. Em 1963, Weinreb et al. já haviam detectado essa mesma molécula em absorção, na direção de Cassiopeia A. Cada um dos estados rotacionais do radical quebra-se em quatro níveis em virtude do desdobramento A e das interações hiperfinas. Dessas interações resultam quatro linhas em rádio, com comprimentos de onda $1=18 \mathrm{~cm}$. A Figura 1 mostra os níveis rotacionais do radical $\mathrm{OH}$.

A segunda espécie molecular detectada como maser foi a de vapor de água $\left(\mathrm{H}_{2} 0\right)$. A sua descoberta deu-se por Cheung et al (1969), na transição em rádio entre dois estados rotacionais excitados e muito próximos em energia (Figura 2).

Em 1974, Snyder e Buhl observaram a emissão maser da molécula de monóxido de silício ( $\mathrm{SiO}$ ), em Orion. Para essa molécula, a emissão maser parece ser proveniente de transições rotacionais envolvendo alguns estadas vibracionais excitados. A Figura 3 mostra alguns níveis rotacionais dessa molécula.

$\mathrm{O}$ reconhecimento observacional de uma fonte maser é importante porque, na Galáxia, ela pode ser encontrada na direção de regiões de formação de estrelas e, também acha-se associada a estrelas do Tipo Espectral M, muito evoluídas (Elitzur, 1982; Reid e Moran, 1988). Assim, o seu estudo pode fornecer importantes informações sobre os estágios inicial e final da evolução estelar.

Uma discussão de fundamental importância para o estudo da emissão maser é a da inversão de população. Como ela aparece na equação de transferência radiativa, a solução desta, em conjunto com as equações que descrevem a distribuição temporal das populações nos níveis maser, fornecem o comportamento da radiação que atravessa a fonte e permitem classificar o seu nível de saturação. O estudo da transferência radiativa em masers aparece em alguns trabalhos importantes e reconhecidos como os de Litvak (1973), Goldreich e Keeley (1972), Kegel (1975), Elitzur (1982), Western e Watson (1984), Alcock e Ross (1985) e Reid e Moran (1988).

Este é o primeiro de uma série de artigos sobre fontes masers. Ele se diferencia dos anteriormente citados pela forma de abordagem e simplicidade com que serão tratados os cálculos envolvendo a transferência radiativa. A Seção 2 mostra a importância da inversão de população e a sua dependência com a equação de transferência radiativa. As soluções desta, para masers não saturados e saturados aparecem na Seção 3 enquanto que as conclusões são mostradas na Seção 4.

\section{2 - INVERSÃO DE POPULAÇÃO E TRANSFERÊNCIA RADIATIVA}

A brilhância da radiação proveniente de um corpo negro que emite à temperatura $\mathrm{T}$ e freqüência $v \hat{e}$ dada pela distribuição de Planck

$$
\mathrm{B}_{\nu}(\mathrm{T})=\frac{2 \mathrm{~h} \nu^{3}}{\mathrm{c}^{2}} \operatorname{exp(h\nu !kT)-1},
$$

* Provém das iniciais de "Microwave Amplification through Stimulated Emission of Radiation". 


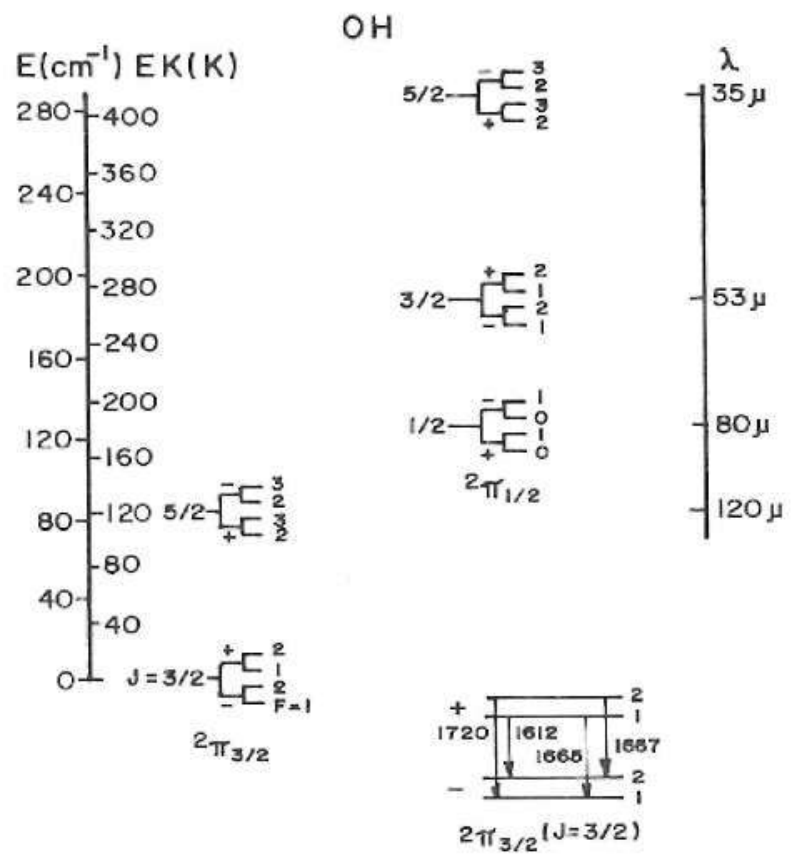

FIGURA 1 - Esquema do espectro rotacional do radical oxidrila $(\mathrm{OH})$. Os desdobramentos $\Lambda_{\theta}$ as divisőes hiperfinas que dividem cada nivel rotacional em quatro subníveis năo estão em escala. $O$ número que aparece ao lado direito de cada nivel de energia representa o momento angular total $\vec{F}$ e inclui o spin nuclear $\vec{l}$. O estado fundamental do radical aparece em destaque em uma escala expandida com as frequencias (em $\mathrm{MHz}$ ) indicadas com flechas.

Fonte: ELITZUR (1982).

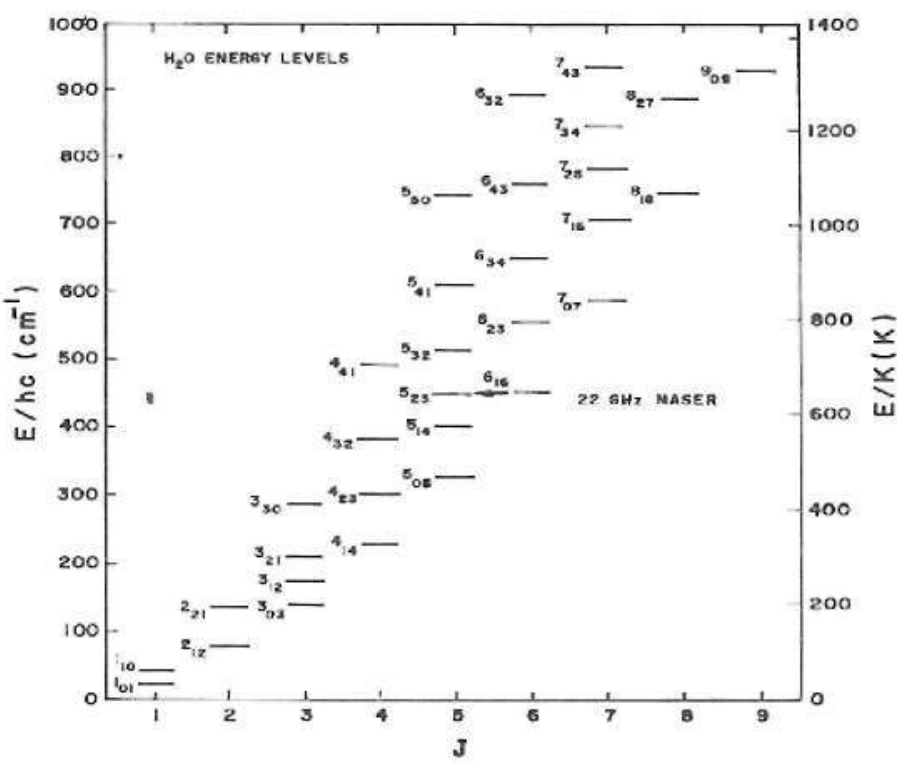

FIGURA 2 - Alguns níveis rotacionais excitados da molécula de vapor de água $\left(\mathrm{H}_{2} \mathrm{O}\right)$ no ramo ortho $\left(\mathrm{K}_{-1}, \mathrm{~K}_{+}\right)=\mathrm{i}, \mathrm{p} \leftrightarrow \mathrm{p}$, f. A transiçăo maser aparece assinalada com unıa flecha entre os niveis $6_{16}-5_{23}$. A figura foi ampliada para destacar a transição maser.

Fonte: DOWNES (1985).

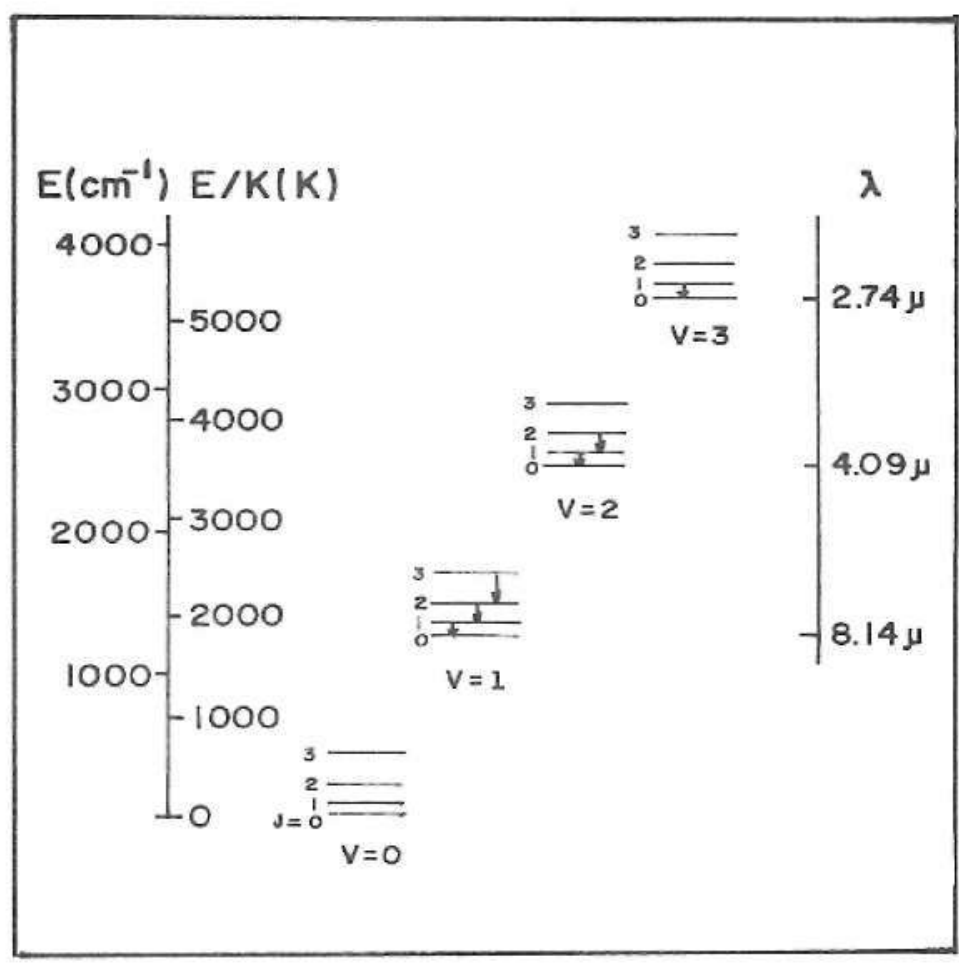

FIGURA 3 - Parte dos niveis de energia rotacional e vibracional da malécula de monóxida de silicio (SiO). As transíçes para as quais as emissós maser foram detectadas aparecem analisadas com flechas.

Fonte: ELITZUR (1982).

onde $h$ é a constante de Planck $\left(\sim 6 \leftarrow 2 \times 10^{-27}\right.$ erg.s $)$ e k é a constante de Boltzmann ( $\left.\sim 1,38 \times 10^{-16} \mathrm{erg} . \mathrm{K}^{-1}\right)$. ̊̀ distribuição fornecida pela Equação 1 pode-se associar a temperatura de brilho, $T_{b}$, da fonte; ou seja, a temperatura de um corpo negro que emite a radiáncia espectral do maser na mesma frequência caracter ística. Portanto,

$I_{\nu}(z)=B_{\nu}\left(T_{b}\right)$

Assume-se neste trabalho, que a tadiação maser seja constituída por ondas eletromagnéticas planas que estao se propa. gando ao longo do eixo $\mathrm{z}$. Tal suposição não tem apenas a finalidade de simplificar a abordagem do problema pois os resultados obtidos por Goldreich e Keeley (1972) e Litvak (1973) sugerem que a radiaçđo proveniente da maioria das fontes é altamente direcional. Daí a razfo de se escrever aqui, a intensidade da radiaçăo em funçăo de uma direção particular.

Para os masers, as temperaturas de brilho sao relativamente altas. Reid e Moran (1988) determinaram valores tao elevados quanto $\sim 10^{B} \mathrm{~K}$ para algumas fontes estelares do radical of e, $\sim 10^{14} \mathrm{~K}$ para alguns masers interestelares de vapor de água. Esses valores foram estimados de fontes relativamente pequenas, com dimensoes angulares que variam entre $10^{-2}$ e $10^{-3}$ segundos de arcos, determinadas por VLBI * (Moran et al, 1968).

$\mathrm{Na}$ faixa espectral em rádio, onde é válida a aproximação de Rayleigh-Jeans, $T_{b}$ e $I_{\nu}(z)$ ainda podem ser re-

* * Provém das iniciais de "Very Long Base-Line Interferometry". 
lacionados mediante a expressåo:

$$
2 \mathrm{k} \nu^{2} \mathrm{~T}_{\mathrm{b}}=\mathrm{c}^{2} \mathrm{l}_{\nu}(\mathrm{z})
$$

Ao atravessar o espaço livre e o meio interestelar (MIE), a radiação apresenta comportamentos distintos. No vácuo, ela praticamente nao sofre qualquer tipo de in. teração. No entanto, ao atravessar o MIE ela é afetada pela emissão e extinçăo (espalhamento + absorçăo) do meio. A equaçăo de transferéncia radiativa descreve o comportamento da radiaçao em seu percurso. Se $z$ medir a distancia percorrida por $I_{\nu}(z)$ e, $k_{\nu}$ for o coeficiente de absorção do meio, entao

$\mathrm{dI},(\mathrm{z})=-\kappa_{\nu} \mathrm{I}_{\nu}(\mathrm{z})+\epsilon_{\nu} \mathrm{dz}$

onde $\epsilon_{\nu}$ será a taxa volumétrica de emissão. A Equaçāo 4 pode também ser escrita como

$\mathrm{dI}_{\nu}(\mathrm{z})=-\mathrm{I}_{\nu}(\mathrm{z})+\mathrm{S}_{\nu} \mathrm{d} \cdot \tau$

onde $\mathrm{d} \tau_{\nu}=\kappa_{\nu} \mathrm{dz} \hat{e}$ o elemento de profundidade ática e $\mathrm{S}_{\nu} \equiv \epsilon_{\nu} / \kappa_{\nu}$ e a razăo entre o "ganho" e a "perda" da radiaçã ou seja, s a funçăo fonte.

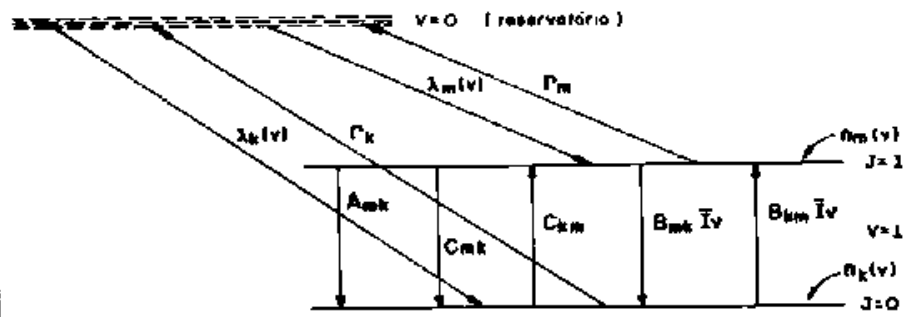

FIGURA 4 - Esquema do modelo maser com dais $n$ iveis de energia e um reservatório de energia. Ver explicações no texto.

Fonte: REID E MORAN (1988).

Em um modelo maser simplificado (Figura 4), contendo os níveis de energia m (superior) e k (inferior) além de um reservatório que teoricamente pode comportar todos os demais níveis rotacionais (Reid e Moran, 1988), os coeficientes que aparecem em (4) stao conhecidos. A emissividade vale (Kegel, 1975)

$\epsilon_{\nu}=\frac{\mathrm{h} \nu \mathrm{A}}{4 \pi} \mathrm{mk}_{\mathrm{e}}(\nu) \mathrm{n}_{\mathrm{m}}(v)$

enquarito que o coeficiente de absorçăo pode ser escrito como

$\kappa_{\nu}=-\frac{\mathrm{h} \nu}{4 \pi} \mathrm{B}_{\mathrm{mk}} \phi_{\mathrm{e}}(\nu) \mathrm{n}_{\mathrm{m}}(\mathrm{v})+\mathrm{h}_{4 \pi} \nu \mathrm{B}_{\mathrm{km}} \phi_{\mathrm{a}}(\nu) \mathrm{n}_{\mathrm{k}}(\mathrm{v})$ onde $A_{\text {tuk }}, B_{m k}$ e $B_{k m}$ sao os coelicientes de Einsteila para os processos de emissão (espontảnea e estimulada) a absorçāo. respectivamente. O coeficiente A mede a probabilidade de ocorréncia da emissao espontánea enquanta que $B$ mede a probabilidade da emissao estimulada (Elitzur. 1982). As densidades volumétricas de população dos níveis maser. por intervalo unitário de velocidade foram representados por $n_{m}(v)$ e $n_{k}(v)$, e os perfis de linha por $v_{e}(v)$ para a emissao. e $\phi_{\mathbf{a}}(\nu)$ para a absorção, respectivamente. O primeiro termo que aparece na Equaçāo 7 representa a emisș̄o estimulada (ou "absorção negativa") enquanto que o segundo, a absorçđ̃o propriamente dita. Assume-se, geralmente (Elitzur, 1982; Reid e Moran, 1988), que as funçőes perfis sđo iguais; ou seja

$\phi_{\mathrm{e}}(\nu)=\phi_{\mathrm{a}}(\nu) \equiv(\nu)$

onde

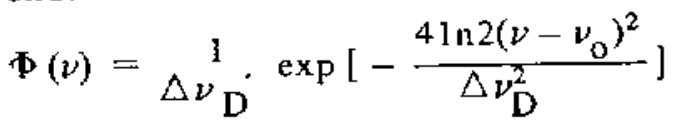

é o perfil gaussiano que leva em consideraç̧o um possível predomínio do movimento térmico entre as moléculas $\mathrm{e}$ $\nu$ e $\nu_{0}$ sao, respectivamente, as frequências de repouso e observada. A largura de linha à meia altura (Efeito Doppler), medida em km $\cdot \mathrm{s}^{-1}$, é dada por Elitzur (1982).

$\Delta \nu_{\mathrm{D}}=0,2\left[2(\ln 2) \frac{\mathrm{KT}}{\mathrm{m}}\right]^{1 / 2}$

onde $\mathrm{m}$ É a peso molecular e $\mathrm{T}$ é a temperatura cinética da fonte.

A condiçāo colocada em (8) é, de certa maneira, questionável porque os efejtos de saturaçăo da linha parecem influenciar diferentemente a distribuiçao das moléculas nos estados envolvidos. No entanto, assumindo-a como verdadeira, a equação de transferència radiativa para a linha de centro de transiçao maser transforma-se em

$$
\begin{gathered}
\mathrm{dI}_{\nu}(\mathrm{z})=\frac{\mathrm{h} \nu}{4 \pi \Delta \nu_{\mathrm{D}}} \quad \mathrm{B}_{\mathrm{km}^{\mathrm{m}}} \mathrm{n}_{\mathrm{m}}(\mathrm{v})\left\{\left[\mathrm{B}_{\mathrm{mk}} \mathrm{n}_{\mathrm{k}}(\mathrm{v})\right] \mathrm{I}_{\nu}(\mathrm{z})+\right. \\
\left.+\mathrm{n}_{\mathrm{m}}(\mathrm{v}) \mathrm{A}_{\mathrm{mk}}\right\} \mathrm{dz}
\end{gathered}
$$

onde o termo da exponencial em (9) foi simplificado. Assumindo identicos pesos estatísticos para os dois níveis da transição maser, a Equaçao 11 resultará em

$$
\mathrm{dI}_{\nu}(\mathrm{z})=\frac{\mathrm{h} \nu}{4 \pi \Delta \nu_{\mathrm{D}}}\left[\Delta \mathrm{n}(\mathrm{v}) \mathrm{B} \mathrm{I} \mathrm{I}_{\nu}(\mathrm{z})+\mathrm{n}_{\mathrm{m}}(\mathrm{v}) \mathrm{A}\right] \mathrm{dz}
$$

onde $\Delta \mathrm{n}(\mathrm{v})=\mathrm{n}_{\mathrm{m}}(\mathrm{v})-\mathrm{n}_{\mathrm{k}}(\mathrm{v})$ e a invers๘̆o de população.

A condição de estado estacionário impőe que o tempo característico para que as condiçðes de bombeamento sofram significativas variaçoes seja maior do que o tempo necessário para que as populaçбes se ajustem a elas (Kegel, 1975). Em outras palavras, isso quer dizer que as variaçōes 
temporais de $n_{m}$ (v) e $n_{k}(v)$ săo praticamente despreziveis. Com o auxílio da figura 4 , tem-se entao. que

$$
\begin{aligned}
\frac{d n_{m}(v)}{d t}= & \lambda_{m 1}(v)\left[n(v)-n_{k m}(v)\right]-\left[n_{m}(v)-n_{k}(v)\right] B I_{\nu}+ \\
& -n_{m}(v) A-n_{m}(v) C+n_{k}(v) C-n_{m}(v) \Gamma= \\
& =n_{m}(v)=0
\end{aligned}
$$

e

$$
\begin{aligned}
\frac{\mathrm{dnl}_{\mathrm{k}}(v)}{\mathrm{dt}}= & \lambda_{\mathrm{k}}(\mathrm{v})\left[\mathrm{n}(\mathrm{v})-\mathrm{n}_{\mathrm{km}}(\mathrm{v})\right]-\left[\mathrm{n}_{\mathrm{k}}(\mathrm{v})-\mathrm{n}_{\mathrm{m}}(\mathrm{v})\right] \mathrm{B} \mathrm{I} \mathrm{I}_{\nu}+ \\
& +\mathrm{n}_{\mathrm{m}}(\mathrm{v}) \mathrm{C}-\mathrm{n}_{\mathrm{k}}(\mathrm{v}) \mathrm{C}-\mathrm{n}_{\mathrm{k}}(\mathrm{v}) \Gamma=\mathrm{n}_{\mathrm{k}}(\mathrm{v})=0
\end{aligned}
$$

onde $n(v)$ é a densidade total de populaçāo,

${ }^{\prime} \mathrm{km}(v)=n_{k}(v)+n_{m}(v)$ s a populaçāo total dos niveis e $\lambda_{m}(v)$ c $\lambda_{k}$ (v) são as taxas de bombeamento do reservatório para os níveis. $\Gamma_{\mathrm{m}}=\Gamma_{\mathrm{k}} \equiv \Gamma$ é a taxa de decaimento (oL perdas) dos níveis para o reservatório e $l_{\nu}$ é a intensidade média da radiaçđo, associada a $\mathbf{I}_{\nu}$ (z) através da relação

$\mathrm{I}_{\nu}=\int \mathrm{I}_{\nu}(\mathrm{z}) \frac{\mathrm{d} \Omega}{4 \pi}=\mathrm{I}_{\nu}(\mathrm{z}) \frac{\Omega}{4 \pi}$,

onde $\Omega \ll 1$ é o pequeno ầngulo sólido do feixe de emissao em microondas da fonte. Segundo Elitzur (1982) e Downes (1985), alguns valores típicos para o coeficiente $A$ sfo: $\mathrm{SiO}(\mathrm{J}=1 \rightarrow 0) \sim 3 \times 10^{-6} \mathrm{~s}^{-1}, \mathrm{H}_{2} \mathrm{O} \sim 2 \times 10^{-9} \mathrm{~s}^{-1} \mathrm{e}$ $\mathrm{OH}$ (estado fundamental) $\sim 10^{-11} \mathrm{~s}^{-1}$. Para a excitaçăo e desexcitaçao colisionais, Downes fomece os valores $\left.\mathrm{C}_{(\mathrm{H}} \mathrm{O}\right)$ $\sim 0^{-2^{-}}$a $1 \mathrm{~s}^{-1}$ enquanto que as taxas de emissfo estimulada para a molécula de vapor de água valem $\sim 10^{3} \mathrm{~s}^{-1}$ $\left(\mathrm{T}_{\mathrm{b}} \sim 10^{13} \mathrm{~K}\right) \mathrm{e} \sim 10^{6} \mathrm{~s}^{-1}\left(\mathrm{~T}_{\mathrm{b}} \sim 10^{16} \mathrm{~K}\right)$. Dessa forma, verifica-se que os valores de A são desprezíveis se comparados aos das demais taxas que aparecem nas Equaçóes 13a e 13b. Levando em considerą̧ão esse fato e utilizando-se (14), estas equaçós podem ser reescritas como

$$
\begin{aligned}
\frac{d n_{m}(v)}{d t}= & n_{m}(v)=\lambda_{m}(v)\left[n(v)-n_{k m}(v)\right]+ \\
& -n_{m}(v) B I_{\nu}(z) \frac{\Omega}{4 \pi}+n_{k}(v) B I_{\nu}(z) \frac{\Omega}{4 \pi}+ \\
& -n_{m}(v) C+n_{k}(v) C-n_{m}(v) \Gamma=0
\end{aligned}
$$

e

$$
\begin{aligned}
\frac{d n_{k}(v)}{d t}= & n_{k}(v)=\lambda_{k}(v)\left[n(v)-n_{k m}(v)\right]+ \\
& -n_{k}(v) B I_{\nu}(z) \frac{\Omega}{4 \pi}+n_{m}(v) B I_{\nu}(z) \frac{\Omega}{4 \pi}+ \\
& -n_{k}(v) C+n_{m}(v) C-n_{k}(v) \Gamma=0
\end{aligned}
$$

Resolvendo-as para a inversao de população, encontra-se que

$\Delta \mathrm{n}(\mathrm{v})=\frac{\mathrm{n}_{\mathrm{km}}(\mathrm{v})\left[\lambda_{\mathrm{m}}(\mathrm{v})-\lambda_{\mathrm{k}}(\mathrm{v})\right]}{\left[\lambda_{\mathrm{m}}(\mathrm{v})+\lambda_{\mathrm{k}}(\mathrm{v})\right]} \frac{\mathrm{F}}{\left[\Gamma+2\left(\mathrm{~B} \mathrm{I}_{\nu}(\mathrm{z}) \frac{\Omega}{4 \pi}+\mathrm{C}\right)\right]}$

de modo que a equação de transfetência radiativa toma, finalmente a forma

$$
\begin{aligned}
\frac{d I_{\nu}(\mathrm{z})}{\mathrm{dz}}= & \frac{\mathrm{h} \nu}{4 \pi \Delta \nu_{\mathrm{D}}}\left\{\frac{\left[\mathrm{n}_{\mathrm{k}}(\mathrm{v})+\mathrm{n}_{\mathrm{m}}(\mathrm{v})\right]\left[\lambda_{\mathrm{m}}(\mathrm{v})-\lambda_{\mathrm{k}}(\mathrm{v})\right]}{\left[\lambda_{\mathrm{m}}(\mathrm{v})+\lambda_{\mathrm{k}}(\mathrm{v})\right]} .\right. \\
& \left.\frac{\Gamma}{\left.\Gamma+2\left(\mathrm{~B} \mathrm{I}_{\nu}(\mathrm{z}) \frac{\Omega}{4 \pi}+\mathrm{C}\right)\right]}\right\} \mathrm{B} \mathrm{I}_{\nu}(\mathrm{z})
\end{aligned}
$$

onde, novamente desprezou-se a taxa de emissão espontâ. nea.

\section{3 - MASERS NAO SATURADOS E SATURADOS}

Nos masers não saturados, as perdas para o reservatório mais as colisøes entre os niveis são superiores a taxa média das transiçôes estimuladas (Downes, 1985). Portanto, a condição física de uma fonte nto saturada é

$\Gamma+2 \mathrm{C}>2 \mathrm{BI}_{v}(\mathrm{z}) \frac{\Omega}{4 \pi}$

de modo que a inversão de população vale

$\Delta n(v)=\frac{\Gamma\left[n_{k}(v)+n_{m}(v)\right]\left[\lambda_{m}(v)-\lambda_{k}(v)\right]}{(\Gamma+2 C)\left[\lambda_{m}(v)+\lambda_{k}(v)\right]}$

Substituindo (19) na Equaçao 7, encontra-se que

$\kappa_{\mathrm{o}}=-\frac{h \nu_{\mathrm{o}} B \Gamma}{4 \pi \Delta \nu_{D}} \frac{\left[\mathrm{n}_{\mathrm{k}}(\mathrm{v})+\mathrm{n}_{\mathrm{m}}(\mathrm{v})\right]\left[\lambda_{\mathrm{m}}(\mathrm{v})-\lambda_{\mathrm{k}}(\mathrm{v})\right]}{(\Gamma+2 \mathrm{C})\left[\lambda_{\mathrm{m}}(v)+\lambda_{\mathrm{k}}(v)\right]}$,

para a transiçâto da linha de centro. Para o caso unidimensional, tomando $\mathrm{I}_{\nu}(\mathrm{z}=0)=\mathrm{I}_{\mathrm{o}}$ como sendo a radiação de entrada no maser e $I_{\nu}(z)=I$, a solução de (17), com $S_{\mathrm{o}}=\epsilon_{\mathrm{o}} / \kappa_{\mathrm{o}}$, será

$I=I_{0} \exp \left(-\kappa_{0} z\right)+S_{0}\left[1-\exp \left(-\kappa_{0} z\right)\right]$ 
para a transição central. Como

$\epsilon_{\mathrm{o}}=\frac{\mathrm{h} \nu_{\mathrm{o}} \mathrm{An} \mathrm{m}_{\mathrm{I}}(\mathrm{v})}{4 \pi \Delta \nu_{\mathrm{D}}}$

entao

$$
\begin{aligned}
I= & I_{0} \exp \left\{\frac{\nu_{0} B \Gamma\left[n_{k}(v)+n_{m}(v)\right]\left[\lambda_{m}(v)-\lambda_{k}(v)\right] z}{4 \pi \Delta \nu_{D}(\Gamma+2 C)\left[\lambda_{k}(v)+\lambda_{m}(v)\right]}\right\}+ \\
& \frac{\Delta n_{m}(v) n_{m}(v)(\Gamma+2 C)\left[\lambda_{k}(v)+\lambda_{m}(v)\right]}{B \Gamma} \frac{\left[n_{m}(v)+n_{k}(v)\right]\left[\lambda_{m}(v)-\lambda_{k}(v)\right]}{\left\{1-\exp \left[\frac{h \nu_{0} B\left[n_{k}(v)+n_{m}(v)\right]\left[\lambda_{m}(v)-\lambda_{k}(v)\right] z}{4 \pi \Delta \nu_{D}(\Gamma+2 C)\left[\lambda_{k}(v)+\lambda_{m}(v)\right]}\right]\right\}}
\end{aligned}
$$

Quando a taxa média da emissão estimulada supera a de decaimento para o reservatório; ou seja, quando as populaçð̄es dos níveis passam a ser controladas pelas transições estimuladas (Downes, 1985), o maser encontra-se saturado. A condição de saturaçăo é, portanto,

$2 \mathrm{~B} \mathrm{I}_{\nu}(\mathrm{z}) \underset{4 \pi}{\Omega} \gg \Gamma+2 \mathrm{C}$.

Neste caso, o coeficiente de absorção também é fornecido por (7) mas a inversao de população passa a valer

$$
{ }_{n}(v)-n_{k}(v)=\frac{\Gamma\left[n_{k}(v)+n_{m}(v)\right]\left[\lambda_{m}(v)-\lambda_{k}(v)\right]}{\left(2 B I \nu(z) \frac{\Omega}{4 \pi}\right)\left[\lambda_{m}(v)+\lambda_{k}(v)\right]},
$$

enquanto que

$$
\kappa_{\nu}=-\frac{\mathrm{h} \nu \Gamma\left[\mathrm{n}_{\mathrm{k}}(\mathrm{v})+\mathrm{n}_{\mathrm{m}}(\mathrm{v})\right]\left[\lambda_{\mathrm{m}}(\mathrm{v})-\lambda_{\mathrm{k}}(\mathrm{v})\right]}{4 \pi \Delta \nu_{\mathrm{D}}\left[2 \mathrm{I}_{\nu}(\mathrm{z}) \frac{\Omega}{4 \pi}\right]\left[\lambda_{\mathrm{m}}(\mathrm{v})+\lambda_{\mathrm{k}}(\mathrm{v})\right]}
$$

e a equação de transferência radiativa vale, portanto,

$$
\mathrm{dI}_{\nu}(\mathrm{z})=\frac{\mathrm{h} \nu \Gamma\left[\mathrm{n}_{\mathrm{k}}(\mathrm{v})+\mathrm{n}_{\mathrm{m}}(\mathrm{v})\right]\left[\lambda_{\mathrm{m}}(\mathrm{v})-\lambda_{\mathrm{k}}(\mathrm{v})\right]}{4 \pi \Delta \nu_{\mathrm{D}}\left[2 \mathrm{I}_{\nu}(\mathrm{z}) \cdot \frac{\Omega}{4 \pi}\right]\left[\lambda_{\mathrm{m}}(\mathrm{v})+\lambda_{\mathrm{k}}(\mathrm{v})\right]}
$$

ou, utilizando-se a aproximação fornecida por Reid e Moran (1988).

$\mathrm{n}_{\mathrm{k}}(\mathrm{v}) \simeq \frac{\mathrm{n}_{\mathrm{k}}(\mathrm{v})+\mathrm{n}_{\mathrm{m}}(\mathrm{v})}{2}$

a equação de transferência valerá:

$$
\underset{\mathrm{dz}}{\mathrm{dl}_{\nu}(\mathrm{z})}=\frac{\mathrm{n}_{\mathrm{k}}(\mathrm{v}) \Gamma \mathrm{h} \nu\left[\lambda_{\mathrm{m}}(\mathrm{v})-\lambda_{\mathrm{k}}(\mathrm{v})\right]}{\Delta \nu_{\mathrm{D}} \Omega\left[\lambda_{\mathrm{m}}(\mathrm{v})+\lambda_{\mathrm{k}}(\mathrm{v})\right]}
$$

cuja solução é

$$
\mathrm{I}_{\nu}(\mathrm{z})=\frac{\Gamma \mathrm{h} \nu \mathrm{n}_{\mathrm{k}}(\mathrm{v})\left[\lambda_{\mathrm{m}}(\mathrm{v})-\lambda_{\mathrm{k}}(\mathrm{v})\right]}{\Delta \nu_{\mathrm{D}} \Omega\left[\lambda_{\mathrm{k}}(\mathrm{v})+\lambda_{\mathrm{m}}(\mathrm{v})\right]} \mathrm{z} .
$$

$\hat{E}$ de se esperar que esse comportamento de $\mathrm{I}_{\nu}(\mathrm{z})$ com z não seja o mesmo considerando-se as demais dimensōes da fonte. De fato, se a forma da fonte maser saturada for equivalente à de uma esfera de raio $r$ ou de $u m$ cilindro longo, com raio $r$ e comprimento $z$, o seu ângulo sólido será inversamente proporcional ao quadrado de z; ou seja, $\Omega \propto(\mathrm{r} / \mathrm{z})^{2}$ (Reid e Moran, 1988). Então, de (27), tem-se que

$\mathrm{dI}_{\nu}(\mathrm{z})=\frac{\mathrm{n}_{\mathrm{k}}(\mathrm{v}) \mathrm{h} \nu \Gamma\left[\lambda_{\mathrm{m}}(\mathrm{v})-\lambda_{\mathrm{k}}(\mathrm{v})\right] \mathrm{z}^{2}}{\pi \mathrm{r}^{2} \Delta \nu_{\mathrm{D}}\left[\lambda_{\mathrm{m}}(\mathrm{v})+\lambda_{\mathrm{k}}(\mathrm{v})\right]} \mathrm{dz}$

que, integrando-se até z' $=\mathrm{z}$, fornecerá

$\mathrm{I}_{\nu}(\mathrm{z})=\mathrm{I}_{\nu}\left(\mathrm{z}_{\mathrm{s}}\right)+\frac{\mathrm{h} \nu \mathrm{n}_{\mathrm{k}}(\mathrm{v}) \Gamma\left[\lambda_{\mathrm{m}}(\mathrm{v})-\lambda_{\mathrm{k}}(\mathrm{v})\right]}{3 \pi \mathrm{r}^{2} \Delta \nu_{\mathrm{D}}\left[\lambda_{\mathrm{m}}(\mathrm{v})+\lambda_{\mathrm{k}}(\mathrm{v})\right]}\left(\mathrm{z}^{3}-\mathrm{z}_{\mathrm{s}}^{3}\right)$,

onde, $z^{\prime}=z_{S}$ é o comprimento da região de saturação da fonte maser.

\section{4 - CONCLUSÕES}

Para o caso de fontes não saturadas, a Equação 20 permite concluir que o coeficiente de absorção para a transição da linha central é invariante; ou seja, mantém-se constante ao longo do percurso da radiação. A profundidade ótica é negativa o que conduz a uma amplificação exponencial na radiação de entrada do maser (Equação 23).

Para as fontes saturadas, o coeficiente de absorção deixa de ser constante e passa a variar com o inverso da intensidade da radiação (Equação 26) e a solução da equação de transferência radiativa permite que se conclua que os eventos de bombeamento são linearmente convertidos em fótons maser. Ocorre então, a produção da radiação ao longo da linha de visada ao invés da amplificação exponencial. Se o ângulo sólido do feixe de radiação proveniente da fonte for constante e a geometria da mesma for plana, a intensidade crescerá linearmente com a distância (Equação 28). No entanto, se o maser saturado se apresentar sob a forma de uma esfera ou cilindro, ela variará com o cubo do comprimento de saturação (Equação 29).

\section{ABSTRACT}

Masers are astrophysical sources in which the absorption coefficient of the corresponding spectral line is negative and the radiation is amplified. For unsaturated sources, the amplification is exponencial. For saturated sources, the mtensity of radiation increases linearly with distance, or with the cube of the path lenght of saturation.

KEY-WORDS: Astrophysical masers: Saturated sources; Unsaturated sources; Radiative transfer; Interstellar medium. 


\section{REFERÊNCIAS BIBLIOGRÁFICAS}

1 - ALCOCK, C.; ROSS, R. R. Saturation and beaming in Astrophysical masers. Astrophys. J. v. 290, p. 433, 1985

2 - CHEUNG, A.C.; RANK, O.M.; TOWNES, C.H.; THORNTON, D.D.; WELCH, W.J. Detection of water in interestellar regions by its microwave radiation. Nature. v. 221, p. 626,1969

3 - DOWNES, D. $\mathbf{H}_{2} \mathrm{O}$ masers in star-forming regions. In: $\mathbf{R}$. Lucas; A. Omont; R. Stora (eds). Birth and Infancy of Stars, p. 557, Elsevier Science Publ., 1983.

4 - ELITEUR, M. Physical characteristics of astronomical masers. Rev. Mod. Phys, v. 54, n. 1, p. 1225, 1982

5 - GOLDREICH, P.; KEELEY, D.A. Astrophysical masers. I. Source size and saturation. Astrophys. J. v. 174, p. $517,1972$.

6 - GUNDERMANN, E.J. Ohservation of the interestellar hydroxyI radical. Ph. D. thesis, Harvard University, Cambridge, Massachusetts, 1965

7 - KEGEL, W.H. Cosmic masers. In: B. Bascheck (ed.), Problems int stellar atmospheres and envelopes, p. 257, Springer-Verlag, Berlin, 1975

8 - LITVAK, M.M. Radiative transport in interestellar masers. Astrophys. J. v. 182, p. 711, 1973
9 -MORAN, J.M.; BURKE, B.F.; BARRETT, A.H.; ROGERS, A.E.E.; BALL, J.A.; CARTER, J.C.; OUDABACK, D.D. The structure of the $\mathrm{OH}$ source in W3. Astrophys. J. (lett.) v. 152, p. L97, 1968

10 - REID, M.J.; MORAN, J.M. Astronomical masers. In: G. Verschuur and K.I. Kellermann (eds.) Galactic and extragalactic radio astronomy, p. 256, Springer-Verlag, 1988

11 - SNYDER, L.E.; BCHL, D. Detection of possible maser emission near 3.48 millimeters from an unidentified molecular species in Orion. Astrophys. J. (lett.) v. 189, p. I. 31,1974

12 - WEAVER, H.; WILLIAMS, D.R.W.; DIETER, N.H.; LUM, W.T. Observations of a trong unidentified microwave line and of emission from the $\mathrm{OH}$ molecule. Nature, v. 208, p. 29, 1965

13 - WEINREB, S.; MEEKS, M.L.; CARTER, J.C.; BARRETT, A.H.; ROGERS, A.E.E. Observations of polarized $\mathrm{OH}$ emission. Nature, v. 208, p. 440, 1965

14 - WESTERN, L.R.; WATSON, W.D. Linear polarization of astronomical masers and magnetic fields. Astrophys. $J$. v. 285 , p. 158,1984

Recebido para publicação em 30/7/90

\section{AGRADECIMENTOS}

* Os autores agradecem os comentários críticos do Dr. J. R.D. Lépine do IAG/USP. Este trabalho foi parcialmente financiado pela CAPES através do Programa PICD/CAPES/UEL. 